$16^{\text {th }}$ International Congress of Metrology, 10003 (2013)

DOI: $10.1051 /$ metrology/201310003

(c) Owned by the authors, published by EDP Sciences, 2013

\title{
A dissolved oxygen calibration bench
}

\author{
Florence Salvetat ${ }^{\mathrm{a}}$, Nolwenn Lamandé, Caroline Le Bihan and Chantal Compère
}

IFREMER, Institut français de recherche pour l'exploitation de la mer, LDCM, Centre de Bretagne, Technopôle de Brest Iroise, 29280 Plouzané, France

\begin{abstract}
This paper presents a metrology bench dedicated to the calibration of optical dissolved oxygen sensors. This set up is used at Ifremer metrology laboratory to calibrate dissolved oxygen sensors integrated on oceanographic probes. The characterization of the bench is seen through the analysis of its stability and its homogeneity. An application is proposed on the calibration of Aanderaa 3835 and 4330 optodes.
\end{abstract}

\section{Introduction}

In oceanography, dissolved oxygen is one of the most monitored parameters. It is, for example, monitored for the assessment of the quality of water, for aquaculture and biodiversity research or for climatology purposes.

In the last decade, a new technology of sensor has been proposed: it is based on an optical measurement of a fluorescence effect.

Since their release, these dissolved oxygen optodes have had a great success in the scientific community. Indeed, they have allowed a wider range of application. For example, they are easier to implement in autonomous high frequency measuring systems. They present some advantages compared to electrochemical sensors: they are easy to handle, easy to maintain and they don't consume any oxygen during measurement. As a consequence, the number of dissolved oxygen optodes integrated on oceanographic systems has highly increased.

But after few years of application, different International and European programs like Argo (ocean observing system based on profiling floats data) or Hypox (in situ monitoring of oxygen depletion associated with hypoxic ecosystems of coastal and open seas, and land-locked water bodies) revealed trueness issues on these optodes. Unfortunately, these issues have not been detected during their calibration. Indeed, till now, the standard calibration protocol carried out on these optodes is based on a two point calibration, one at full saturation $(100 \%)$ and the other one at $0 \%$. This practice comes from the ease to perform these two points: the $0 \%$ is done by a sodium bisulfite solution and the $100 \%$ by stirring water in an open tank.

But this protocol presents several limitations:

It assumes that optodes signals are linear, which is not the case.

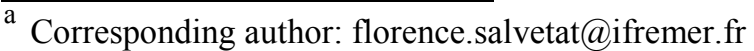

- It doesn't check the full range measurement of the optodes which can reach more than $100 \%$ saturation in oversaturated areas.

- It doesn't include the temperature effect on the dissolved oxygen concentration measurement expressed in $\mu \mathrm{mol} / 1$.

In order to improve the calibration protocol and also to investigate these trueness issues, the metrology laboratory of Ifremer developed in 2008 a dissolved oxygen calibration bench. This bench is able to perform different dissolved oxygen concentrations in the range from $0 \mu \mathrm{mol} / 1$ to oversaturation. It also allows calibrations at different temperatures and different salinities. To complete the protocol, sensors results are compared to Winkler analysis as reference measurements [1].

We propose in this document to present this calibration bench:

- Its characterization will be discussed through the analysis of its stability and homogeneity.

- $\quad$ The different issues will be described.

- $\quad$ Finally, the bench ability for calibration will be seen through 3835 and 4330 Aanderaa optode calibrations.

\section{Description of the bench}

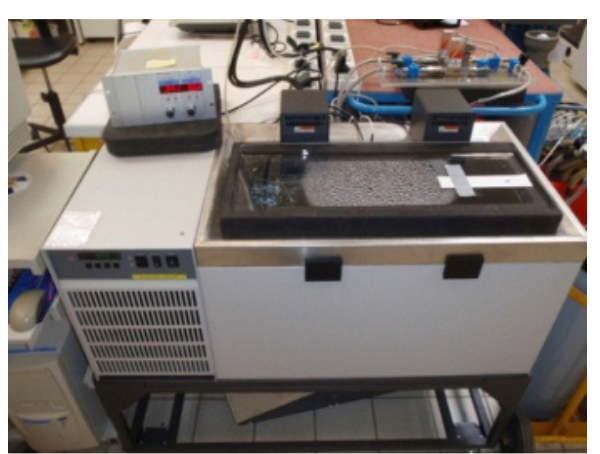

Figure 1: Dissolved oxygen calibration bench 
The bench is made of a regulated temperature bath and a bubbling system injecting simultaneously nitrogen and oxygen with mass flow meters/controllers.

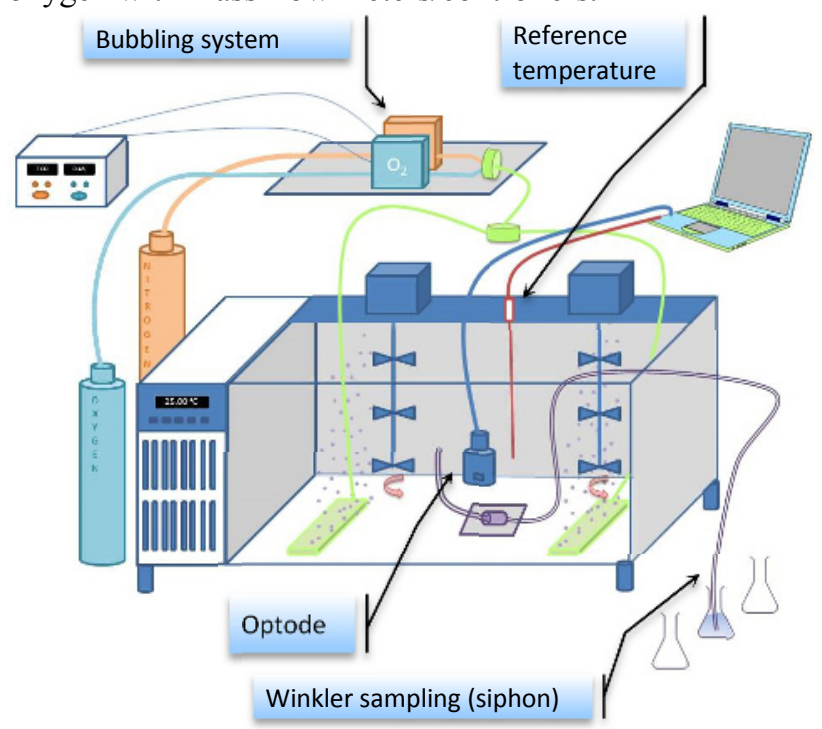

Figure 2: Drawing of an optode calibration in the bench

\subsection{The temperature regulated bath}

The bath is wide enough to include several sensors (110 litres).

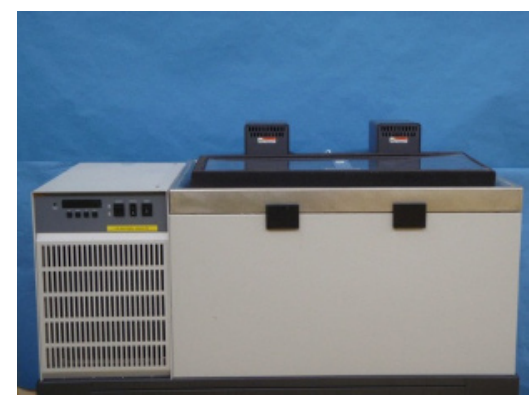

Figure 3: Temperature regulated bath

It is used to regulate temperature from $-1.5^{\circ} \mathrm{C}$ up to $+40^{\circ} \mathrm{C}$, which is the ordinary oceanographic range. Its temperature stability is $0.001^{\circ} \mathrm{C}$ and its homogeneity is $0.005^{\circ} \mathrm{C}$. This bath can either be filled with fresh water, seawater or a mixture of both.

\subsection{The bubbling system}

We set up a system in such a way as to reproduce the natural exchange of oxygen between air and water. That is why we chose to bubble oxygen and nitrogen in the water.

The bubbling is done with mass flow meters/controllers to ensure the homogeneity of the gas mixture.

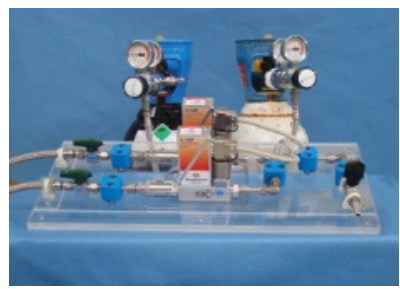

Figure 4: Mass flow meters/controllers

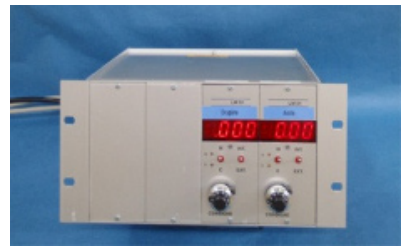

Figure 5: Power supply and readout system

The gas mixture is injected through aquarium air stones to favour the exchange between gas and water.
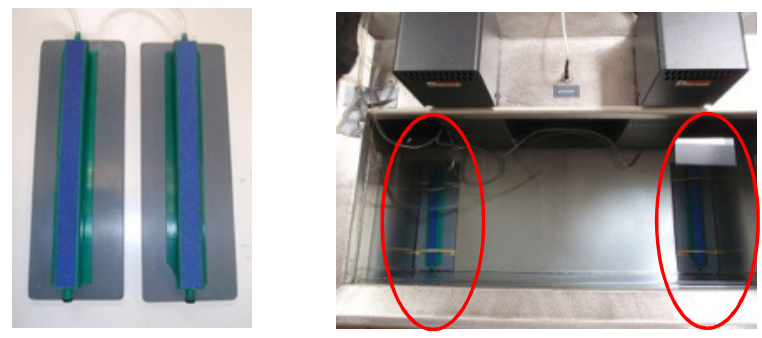

Figure 6: Aquarium air stones

\section{Characterization of the bench}

In order to qualify the bench, we performed a characterization in dissolved oxygen concentration similar to the characterization in temperature.

We thus studied the stability over time of the concentration in dissolved oxygen and its spatial homogeneity.

The requirements to validate the bench are to reach stabilities and homogeneities of a few micromoles per litre. Indeed, optode specifications usually read accuracies of a few micromoles per litre and Winkler measurements uncertainties are around $4 \mu \mathrm{mol} / 1$.

\subsection{Bench stability}

The stability of the bench was monitored using a dissolved oxygen optode placed in the centre of the bath. The resolution of the optode is lower than $1 \mu \mathrm{mol} / 1$.

Several tests were performed at different temperatures (from $0^{\circ} \mathrm{C}$ up to $30^{\circ} \mathrm{C}$ ) and dissolved oxygen percent saturations (from $0 \%$ up to $140 \%$ ). These tests match the following concentration range: $0 \mu \mathrm{mol} / 1$ to $400 \mu \mathrm{mol} / 1$. Some examples of recordings are given in figures 7 and 8 below. 


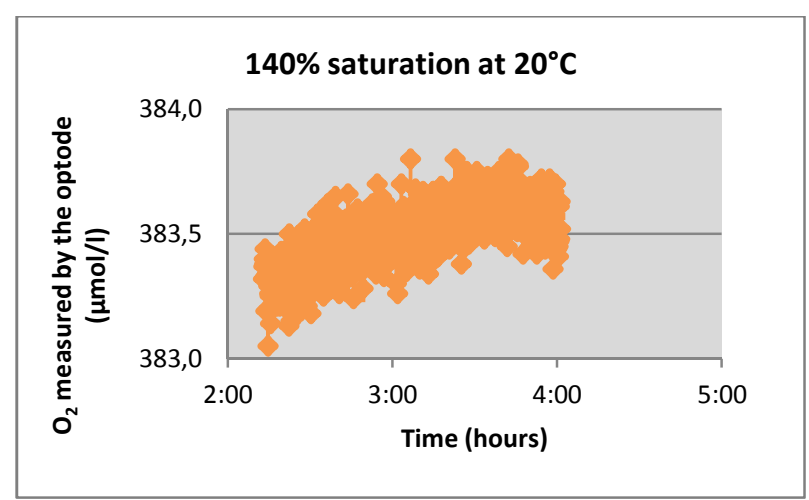

Figure 7: Optode recording around $140 \%$ saturation at $20^{\circ} \mathrm{C}$

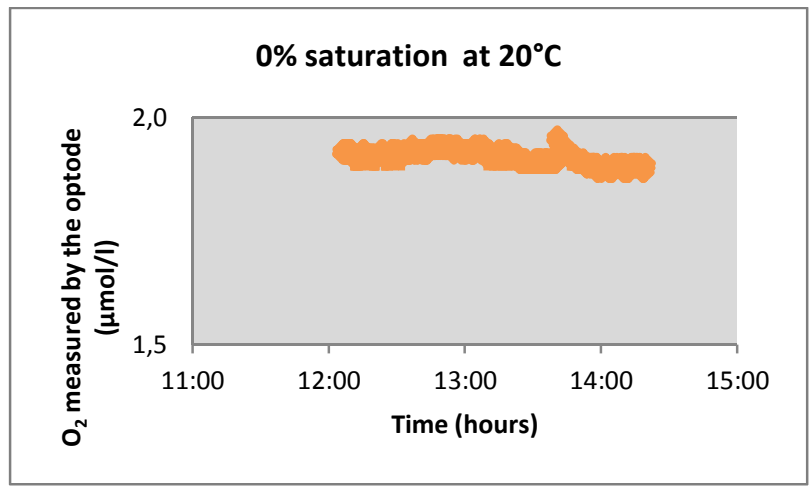

Figure 8: Optode recording around $0 \%$ saturation at $20^{\circ} \mathrm{C}$

The results showed stabilities lower than $0.5 \mu \mathrm{mol} / 1$ within 1 hour which is totally in agreement with the requirements. We even obtained steady states lasting more than 10 hours at both low and high concentrations.

These experiments also revealed:

- the time needed to reach stability after modification of the set point: 3 to 4 hours for a decrease in concentration from $400 \mu \mathrm{mol} / 1$ to $250 \mu \mathrm{mol} / 1$ when the flow of nitrogen is set at $31 / \mathrm{min}$.

- $\quad$ the ability of the bench to reach concentrations near $0 \mu \mathrm{mol} / \mathrm{l}$ : the lowest concentration achieved measured by Winkler analysis is $5 \mu \mathrm{mol} / 1$ but this value depends on the airtightness of the bath (see paragraph 4.1),

- the similarity of the stability between concentrations at $100 \%$ made with the bubbling system and made with a stirring bath: in both cases, stability is lower than $0.5 \mu \mathrm{mol} / 1$ within 1 hour.

\subsection{Bench homogeneity}

The homogeneity of the bench was determined by comparing Winkler samplings in different places of the bath. The drawing of the test is given in figure 9 below.

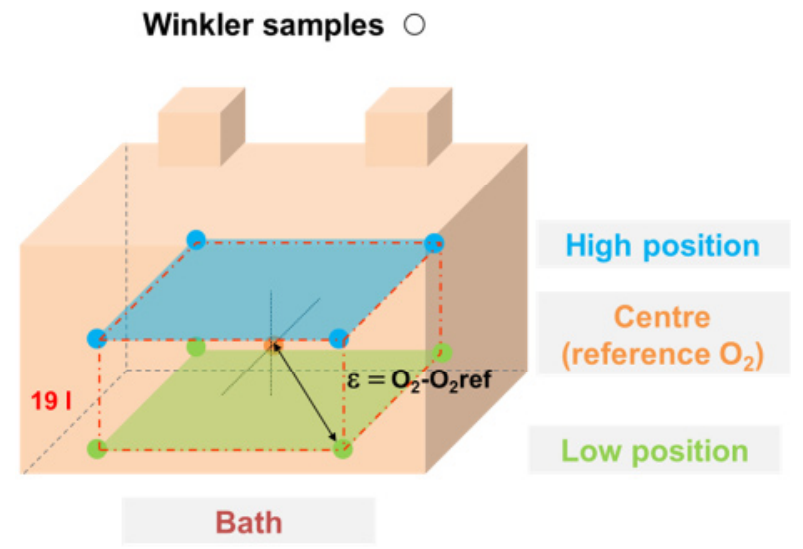

Figure 9: Drawing of the homogeneity protocol

The homogeneity $\varepsilon$ was obtained by calculating the discrepancy between the Winkler value in the corners of the bath and the Winkler value in the centre of the bath.

Several concentrations were tested and homogeneity didn't exceed $2 \mu \mathrm{mol} / 1$. These results are in accordance with the requirements.

\section{The issues}

\subsection{Airtightness of the bath}

The airtightness of the bath cover is critical:

- $\quad$ to obtain the stability of the concentration,

- $\quad$ to reach concentrations as close as possible to the set point,

- $\quad$ to reach the $0 \mu \mathrm{mol} / \mathrm{l}$ concentration.

However, getting an efficient airtightness is really complex, especially when many cables of sensors and the sampling tube get through the cover.

\subsection{Sampling effect on the concentration}

The sampling for the Winkler analysis is performed by siphoning the water of the bath. As we can see in figure 10 , from time to time, this sampling can disturb the stability of the concentration. The effect is either a quick decrease or increase of the concentration with no link with the value of dissolved oxygen concentration in the bath.

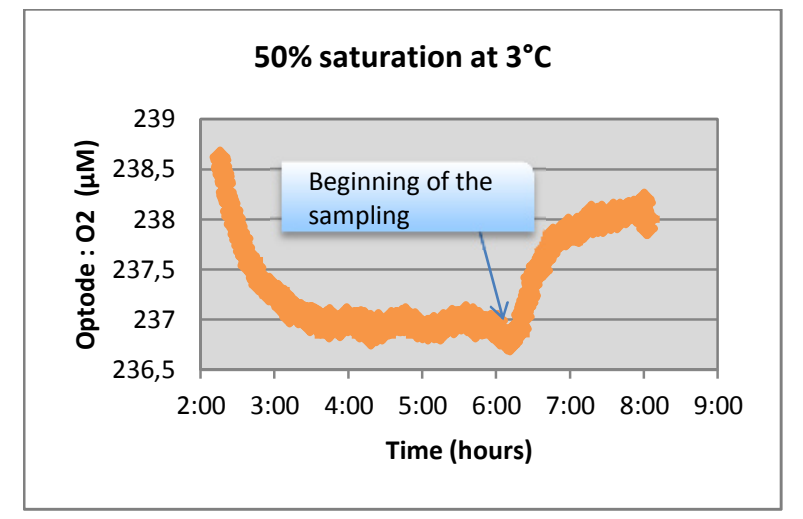

Figure 10: Winkler sampling effect 


\section{Examples of calibrations}

Several calibrations have been performed in the bench. Here are two examples of calibration on two types of optodes from the manufacturer Aanderaa.

\subsection{Calibration protocol}

The calibration is performed in fresh water at different temperatures $\left(0,10,15,20\right.$, and $\left.30^{\circ} \mathrm{C}\right)$. For each temperature (except $15^{\circ} \mathrm{C}$ ), we perform calibration at 0 , 20, 100 and $135 \%$ of dissolved oxygen. For some concentrations, we repeat two to three times the experiment.

Previously, we have checked that there was no hysteresis effect on the optode.

If needed, the optode is adjusted following the SternVolmer equation proposed by Uchida et al. [2].

\subsection{Calibration of a 4330 Aanderaa optode}

The calibration results are summarized on the figure 11 and on the figure 12 (zoom of the figure 11). The correction to apply to the optode is plotted as a function of the dissolved oxygen concentration measured by Winkler analysis.

The graph presents:

the corrections before adjustment of the optode for several temperatures,

the corrections after adjustment of the optode.

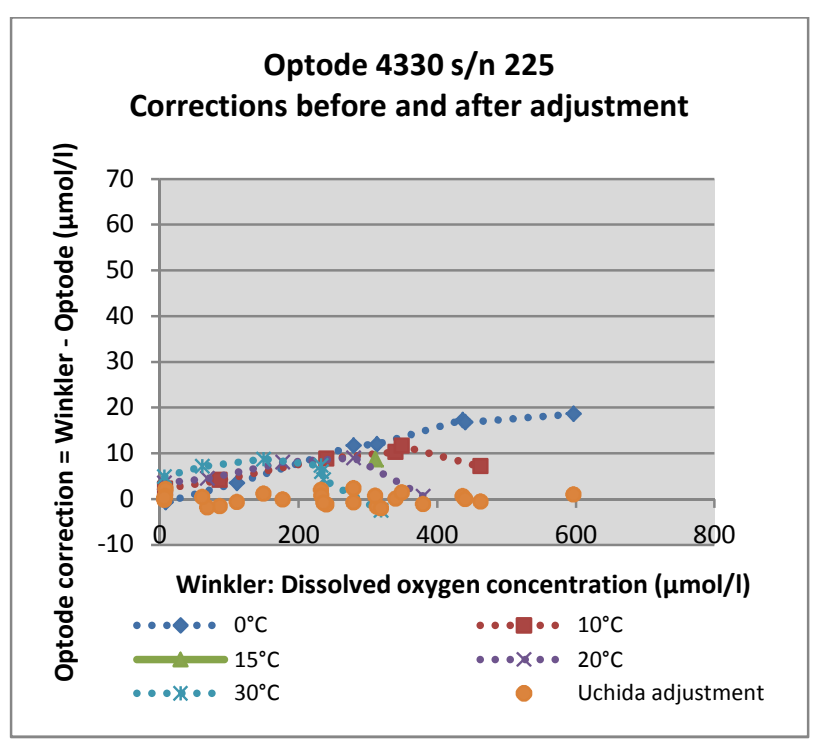

Figure 11: Calibration of a 4330 Aanderaa optode

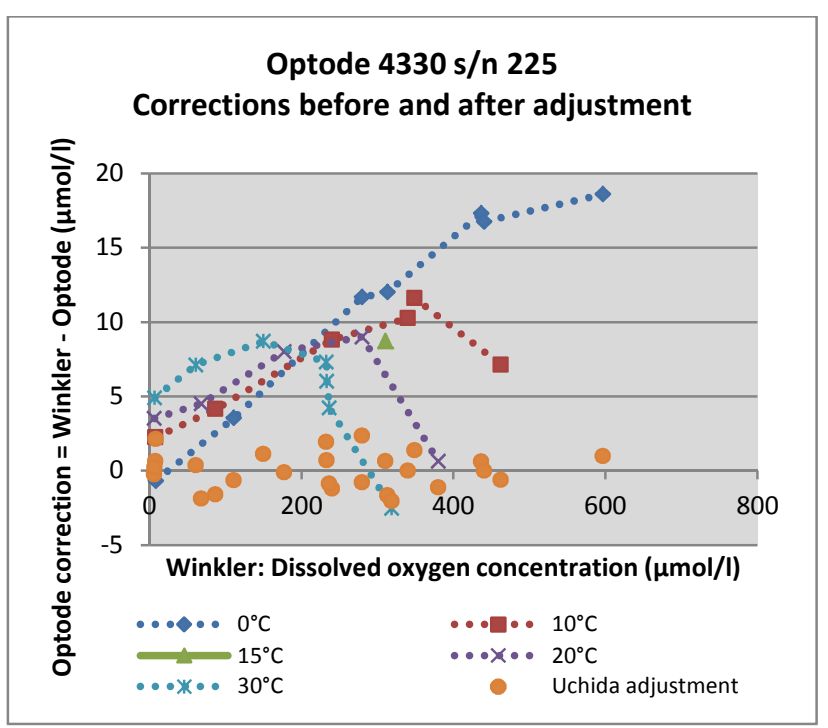

Figure 12: Calibration of a 4330 Aanderaa optode - Zoom of figure 11

The results lead to several comments.

At first, we can see for each temperature a drop of the correction for the higher concentration. This concentration corresponds to the $135 \%$ point which is not calibrated by the manufacturer. A consequence is a loss of linearity of the sensor. With the traditional calibration protocol $(0 \%$ and $100 \%)$, we would have not detected this modification in the trend of the trueness errors.

Then, the effect of temperature on the sensor appears clearly. It can leads to errors around $5 \mu \mathrm{mol} / 1$ in the range $0-100 \%$ and greater than $10 \mu \mathrm{mol} / 1$ beyond $100 \%$. These errors beyond $100 \%$ are higher than the manufacturer specifications.

Finally, the adjustment recommended by Uchida et al. [2] fits perfectly the behaviour of the sensor. The residuals don't exceed $3 \mu \mathrm{mol} / 1$ which is in agreement with the manufacturer specifications and the scientific expectations.

\subsection{Calibration of a 3830 Aanderaa optode}

The same calibration is carried out on another type of optode of the same manufacturer. The graph in figure 13 presents the results of the calibration. The labels are the same as the previous graphs. 


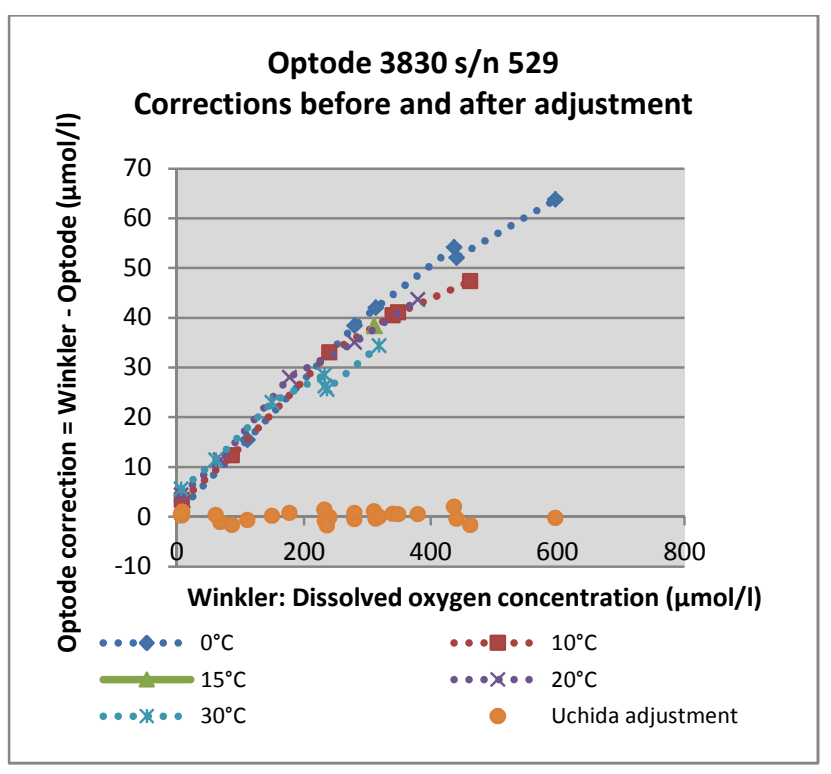

Figure 13: Calibration of a 3830 Aanderaa optode

In this calibration, we don't notice such an important drop of the correction for the concentration corresponding to $135 \%$ (see graph 11 with the same scale). However, the concentration discrepancy due to temperature and the lack of linearity of the sensor (several $\mu \mathrm{mol} / \mathrm{l}$ ) are of the same order of concentration as the accuracy given by the manufacturer. Then again, the adjustment recommended by Uchida et al. [2] corrects properly the data.

\section{Conclusions}

Through the calibration experiments presented, we have seen clearly the need to improve the former calibration protocol of dissolved oxygen optodes. It is absolutely necessary to include in the protocol, calibration at several temperatures and at several dissolved oxygen concentrations covering the full range of measurement of the sensor.

In consequence, calibration laboratories must own a setup which allows performing this multi-point calibration procedure. Different systems were built by metrology laboratories: some are bubbling equipment, others are based on electrochemical principles [3]. Currently, an inter-laboratory comparison is held in the framework of the Argo program in order to compare the different systems used by the oceanographic institutes all over the world to calibrate their dissolved oxygen sensors.

Finally, further influence parameters need to be studied. The pressure has already been identified by Uchida et al. [2]. But calibrating optodes under pressure is a tricky procedure and is not yet in the habits. It would be necessary to develop and propose a pressure setup. Salinity needs also to be investigated to quantify its impact in terms of correction and uncertainty on the optode response.

\section{References}

1 A. Aminot, R. Kerouel, Hydrologie des écosystèmes marins Paramètres et analyses, Chap. VII (Ifremer, 2004)

2 H. Uchida, T. Kawano, I. Kaneko, M. Fukasawa, J. Atmos. Oceanic Technol., 25, 2271-2281 (2008)

3 H.C. Bittig, B. Fiedler, T. Steinhoff, A. Körtzinger, Limnol. Oceanogr. Methods 10:921-933 (2012) 